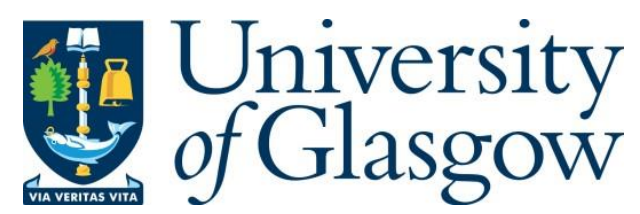

Dhimish, M. and Kettle, J. (2021) Impact of solar cell cracks caused during potentialinduced degradation (PID) tests. IEEE Transactions on Electron Devices, (doi: 10.1109/TED.2021.3135365).

There may be differences between this version and the published version. You are advised to consult the publisher's version if you wish to cite from it.

https://eprints.gla.ac.uk/261022/

Deposited on: 15 December 2021

Enlighten - Research publications by members of the University of Glasgow https://eprints.gla.ac.uk 


\title{
Impact of Solar Cell Cracks Caused During Potential Induced Degradation (PID) Tests
}

\author{
Mahmoud Dhimish, Member, IEEE, Jeff Kettle, Member, IEEE
}

\begin{abstract}
Potential-induced degradation (PID) of photovoltaic (PV) modules is one of the most severe types of degradation in modern modules, where power losses depend on the strength of the electric field, the temperature and relative humidity and the PV module materials. Understanding how module defects impact PID is key to reducing the issue. This work investigates the impact of cracks and fractural defects in solar cells and their impact on PID. We have tested using electroluminescence (EL) imaging how solar cells with varying levels of cracks/defects impact PID and our results indicate that such defects have a sizeable influence on the PID. We also confirmed the strong relationship between the size of the initial defect/crack and the presence of hotspots. As the crack (dark) area increased, there was a further increase in the cell's temperature under standard test conditions. In this work, it is observed that minor cracks in solar cells have no considerable PID effect for the solar cells providing some reassurance that quality control can reduce this degradation pathway.
\end{abstract}

Index Terms-Solar cells; PID, cracks, electroluminescence, performance analysis.

\section{INTRODUCTION}

$\mathrm{O}$ NE of the most valuable attributes of solar cell technology is its high stability, with operational lifetimes of over 30 years. Nevertheless, there are numerous degradation mechanisms, and these collectively reduce the module output power over time. One of the main degradation mechanisms is called potential-inducted-degradation (PID) [13]. For many photovoltaic (PV) systems, PID is one of the leading causes of module degradation and is caused by voltage, as well as the interaction of this stress factor with temperature and humidity. PID occurs when the module's voltage potential and leakage current drive ion mobility within the module between the semiconductor material and other components such as the glass, frame, or mount [4]. PID becomes more prevalent as the module ages, and whilst it doesn't affect all modules, it does have a severe impact as it cannot be fixed [5].

In a recent study, [6], a PV module subjected to a reverse bias of $160 \mathrm{~V}$ voltage was shown to exhibit severe PID, leading to the formation of hotspots which appeared on the

M. Dhimish is with the Department of Electronic Engineering, University of York, York Y010 5DD, UK. (e-mail: m.a.dhimish@hud.ac.uk).

J. Kettle is with James Watt School of Engineering, University of Glasgow, Glasgow G12 8QQ, UK. (e-mail: Jeff.Kettle@glasgow.ac.uk).

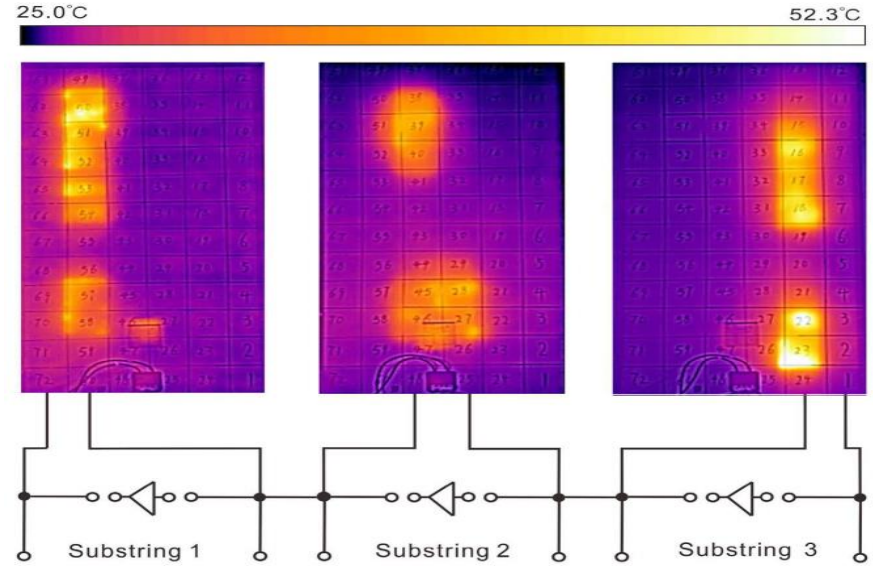

Fig. 1. Thermographic image of three substrings of module 3 under reverse $160 \mathrm{~V}$ bias [6].

modules, as presented in Fig. 1 for three different PV modules connected in series. This study showed that the PID resulted in a reduction of the current density and the open-circuit voltage. Improved testing has been the focus of much recent research; for example, in [7], authors have presented an approach to test the reversible and irreversible PID testing to bifacial PERC solar cells. Other researchers $[8,9]$ have reported that cracks in solar cells can accelerate PID due to the localized heat caused by the cracks. This work was supported by studies conducted using electroluminescence (EL) imaging before and after PID testing. This affirmed that cracks could lead to hotspots in the solar cells, and as a result, PID is expected, yet there were no discussions on the type/size of cracks and their expected impact upon PID.

To prevent PID on a solar cell level, one research direction is to optimize the top surface, for example, the antireflection coating (ARC) [10-12]. Recent studies show that the thin silicon dioxide ( $\mathrm{SiO} 2)$ ARC layer combined with n-type and p-type solar cells can reduce PID in solar cells. However, this coating layer cannot prevent cracks and structural defects forming, but perhaps mitigate for the impact of PDI on that particular cell. Regardless of the impact of the ARC, at a PV module level, every component must be resistant to PID; including the encapsulants, absorption layers, and preferably the glass as small leakage currents can cause ion migration.

the glass as small leakage currents can cause ion migration.

In Fig. 2, the EL image of solar cells affected by the typical cracks and/or structural defects is presented. These have been categorized into 3 groups by these authors; in Fig. 2(a), the cell is affected by minor cracks, whereas Fig. 2(b) shows that the cell has a dark area on the bottom edge. The last image, Fig. 2(c), presents a solar cell affected by a breakdown area. 


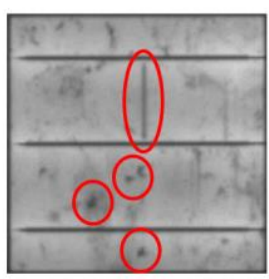

(a)

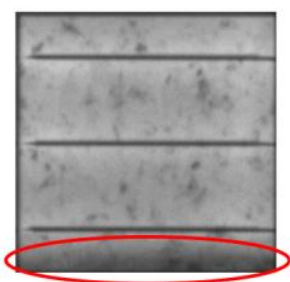

(b)

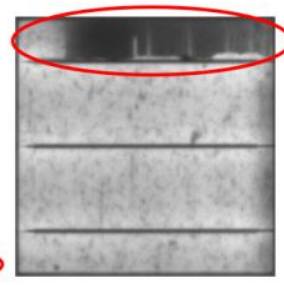

(c)
Fig. 2. PV module affected by cracks or structural defect. (a) Minor cracks, (b) Dark area, (c) Breakdown the area.

These types of cracks have been previously reported in studies such as [13-16], showing that cracks can lead to a drop in the efficiency of the solar cells. However, there has been no explanation of the behavior of these cracks on the actual current density and the potential impact upon PID.

In this paper, the relationship between cell cracking and PID in c-Si solar cells is examined. The analysis will include the short circuit current density map of cracked solar cell samples, PID test results, and more specific instances to use a scanning electron microscope to further investigate the crack distributions.

This paper is organised as follows: Section II comprises the sample preparation and the EL and PID testing setups. In Section III, we present the results of the tested solar cell samples. Section IV presents the output power measurements and finally, Section V presents the conclusions of the complete study.

\section{Samples Preparation ANd Experimental Procedure}

In this work, several c-Si solar cells affected by different types of cracks have been investigated. The solar cells were dismounted from a PV module operated in a field condition for five years (Fig. 4(a)); Solar UK ltd handled this process. The solar cells comprise three busbars and at standard test conditions (STC), and as provided by the manufacturer datasheets, the cells' short circuit current density $J_{s c}$ is equal to $38.8 \mathrm{~mA} / \mathrm{cm}^{2}$, open-circuit voltage $V_{o c}$ is equal to $0.61 \mathrm{~V}$, and the peak power is $4.72 \pm 2 \% \mathrm{~W}$.

Initially, we have used the EL imaging setup shown in Fig. 3. The EL comprises a digital single-lens reflex camera with a resolution of $6 \mathrm{k} \mathrm{x} 4 \mathrm{k}$ pixels, and the filter is removed and calibrated to allow sensitivity to electroluminescence picture (peak wavelength $1150 \mathrm{~nm}$ ). The camera lens is $18-55 \mathrm{~mm}$, and a programmable $15 \mathrm{~A}, 60 \mathrm{~V}$ power supply is available to connect with the solar cell. To capture the finest EL images, the solar cells were connected to a power supply under $I_{s c}$ current $(8.07 \mathrm{~A})$.
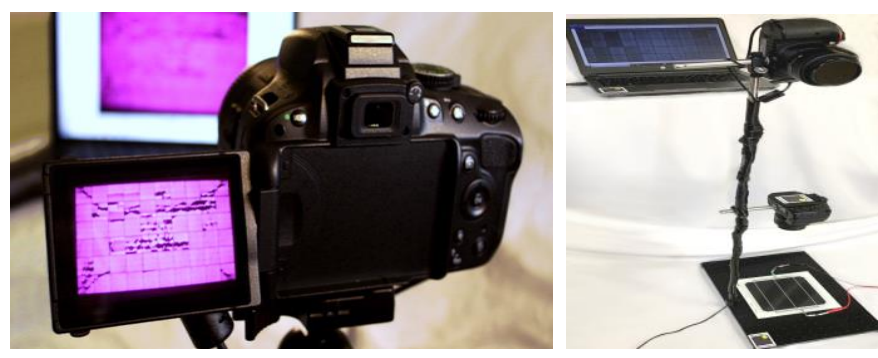

Fig. 3. EL imaging setup
We have examined several solar cell samples categorizing different cracks shapes on distribution as well as structural defects. The EL image of the tested cells is shown in Fig. 4. According to Fig. 4(b), the solar cell sample (\#1) is affected by minor defects/cracks across the cell. It was evident by previous research that such cracks do not reduce the output power of the solar cell. While solar cell samples (\#2 and \#3) are affected by cracked areas along with dark areas (labelled by the triangle), this type of crack has not been fully understood, and there is no information on whether it could lead to PID in the solar cell.

The last solar cell sample (\#4) is affected by a breakdown region (labelled by the triangle). There are some proposals in the literature on whether this area can lead to PID or breakdown in the busbar; however, experimentation results have not yet been proclaimed. Hence, our results on examining these defects in the solar cells would significantly contribute to understanding the variations of the cracks along with their relationship with solar cell PID.

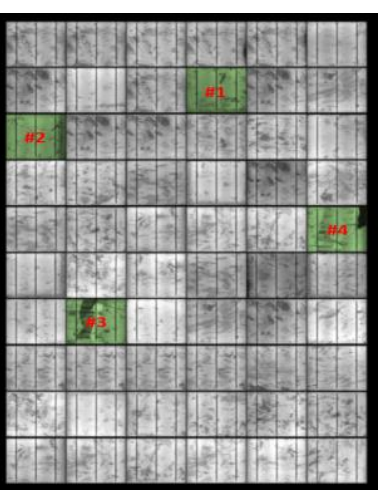

(a)
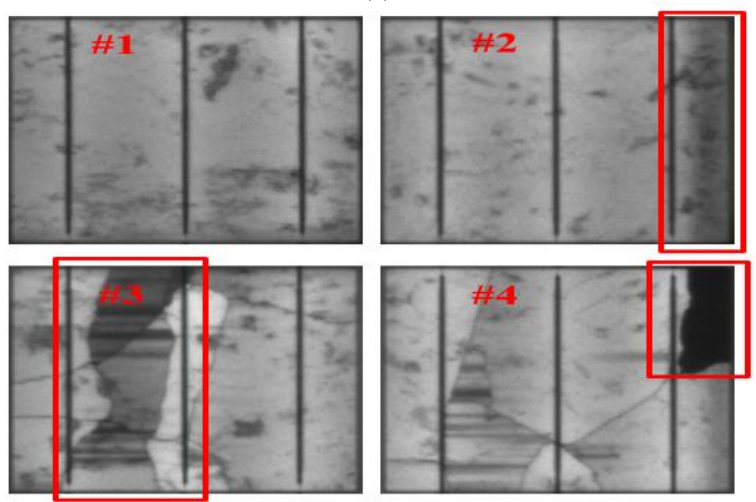

(b)

Fig. 4. (a) EL image of the PV module before the dismounting process, (b) After the dismounting process, the EL image of the tested solar cell samples, the first sample (\#1), has a minor defect/crack across the cell. In contrast, samples (\#2 and \#3) are affected by a dark area with additional cracks in the cell. The last sample (\#4) is affected by a breakdown region, as evidence by the solid black area.

The PID test of the solar cell samples was conducted using the PIDcon instrument made by Freiburg Instruments $\mathrm{GmbH}$ in Germany and is shown in Fig. 5(a). The PID test's basic setup (Fig. 5(b)) comprises an aluminium foil placed on the front glass, covering the whole solar cell. The positive terminal of the PIDcon power supply is applied to the aluminium foil. The negative terminal has been applied to the front contact of the solar cell. We use the standard PID testing 
conditions for this work, where the temperature is set at $85^{\circ} \mathrm{C}$, the voltage is set to negative $1000 \mathrm{~V}$, and the PID test is conducted for 96 hours. The same procedure was performed on all four solar cell samples to investigate how the differing crack distributions were affected by PID testing.

In addition, we have tested the solar cell samples under a scanning electron microscope (SEM); the SEM is shown in Fig. 5(c). The SEM is interfaced with a personal computer for data acquisition. The Back Scattered (Everhart-Thornley detector (ETD)) was selected to observe the impact of the cracks on the actual micro/nanostructure of the solar cell samples.

Laser beam induced current (LBIC) mapping was also used to observe changes in the $J_{s c}$ for the tested solar cells. The LBIC is non-destructive optical testing for the characterization of solar cells. This technique uses a light source from laser diodes of different wavelengths between 638 to $850 \mathrm{~nm}$. An electrical bias on the laser is modulated to produce the pulsed laser beam. We have used this technique because it provides an output image which contrasts from the EL technique and provides an alternative non-destructive view of the changes that are occur spatially within the solar cell. The LBIC provides greater detailed mapping of the cells' surface and bulk photogeneration properties, along with the actual JSC current, which, can offer a complementary map of the cell's appearance, particularly for the cracked area. In contrast, the EL gives an image showing high resolution images of solar cell fractures and cracks, as well as carrier injection into different regions of the solar cell. Undertaking LBIC in conjunction with EL measurements provides a fuller understanding of the changes during testing.

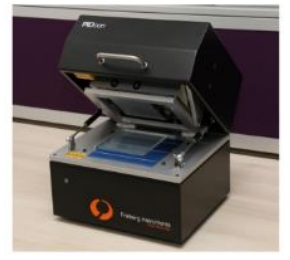

(a)

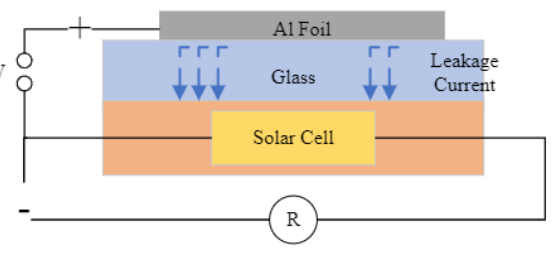

(b)

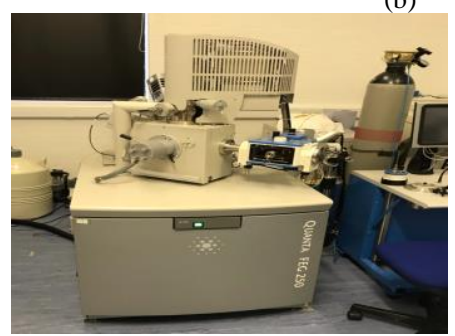

(c)

Fig. 5. (a) PID (PIDcon) instrument, (b) PID testing setup, (c) SEM (Quanta FEC250) testing facility.

\section{Results}

The first solar cell sample (EL image shown in Fig. 4, \#1) was observed before and after the PID test. This solar cell possessed only minor defects/cracks across its surface. We investigated the short circuit current density, as the drop in the open-circuit voltage is not significant during the testing response (fraction of $10 \mathrm{mV}$ ) is expected to drop [17, 18] after the PID testing is performed.
The image of the $J_{s c}$ before and after the PID test is shown in Fig. 6(a) and Fig. 6(b), respectively. The difference between the before/after images is shown in Fig. 6(c). The most significant defects/cracks in the solar cell sees the biggest drop in $J_{s c}$. For example, on, the area labelled by the circle has no losses in the $J_{s c}, 38 \mathrm{~mA} / \mathrm{cm}^{2}$. However, for the cracked/fractured area, labelled by the rectangle, the $J_{s c}$ drop is approximately equal to $-10 \mathrm{~mA} / \mathrm{cm}^{2}(27 \%$ relative reduction). This would correspond to a hotspot in an operational PV module, potentially leading to greater module defects as time progressed.

Following the observation of sample \#1, minor cracked/defective areas in a solar cell are unlikely to cause a major and immediate PID. This consequence was concluded by observing that the pattern of Fig. 6(a) and Fig. 6(b) are almost equal. There are moderate differences between the $J_{s c}$ before and after the PID, as shown in Fig. 6(c). We have labelled the key areas that exhibit a reduction in (leakage) current after the PID testing was completed. The blue regions in Fig. 6(c) correspond to a negligible change in degradation (no difference in the $J_{S C}$ ).

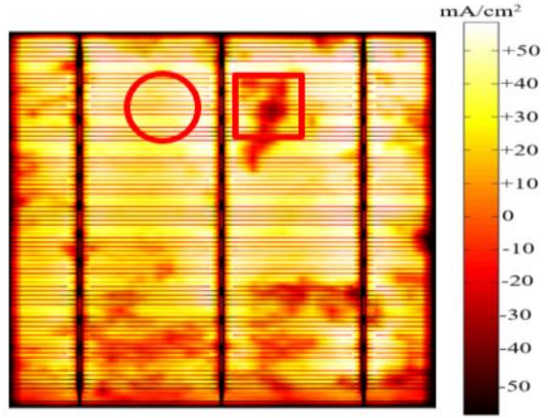

(a)

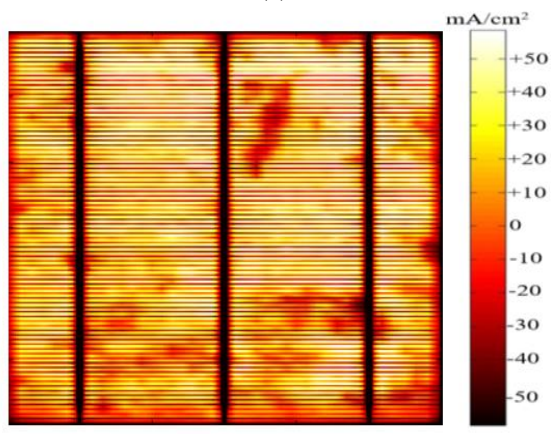

(b)

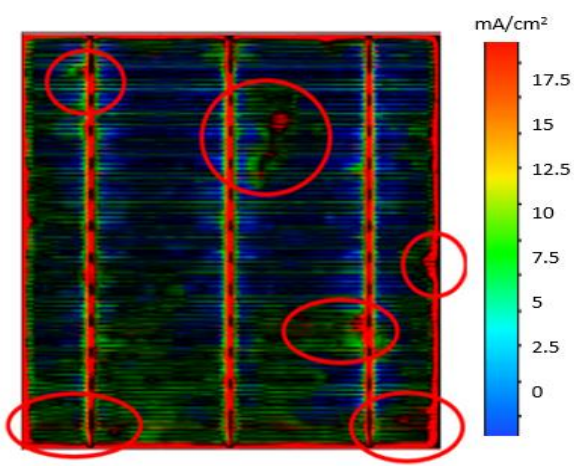

(c)

Fig. 6. $J_{s c}$ for Solar cell \#1. (a) before the PID test, (b) after the PID is completed, (c) Difference in the $J_{s c}$ before and after the PID test. 
Now consider the behavior of solar cell sample \#2, where the LBIC images before the PID test began is shown in Fig. 7(a). The dark area on the right-hand side of the solar cell clearly exhibits a drop in the $J_{s c}$. In agreement with the earlier data, the $J_{s c}$ reduced significantly after the PID test was completed (Fig. 7(b)). Remarkably, the images indicate that the dark area is likely to be causing a more severe PID to the cell. There is a noticeable expansion of the dark regions across the right-hand side where the original EL image has an initial dark area.

The difference between the $J_{s c}$ before and after the PID is shown in Fig. 7(c). There is a significant drop in the current on the right-hand side of the solar cell, which is the same area that had the initial shading/defect. This confirms that such dark areas in the original EL images can be classified as highrisk regions for PID degradation in solar cells.

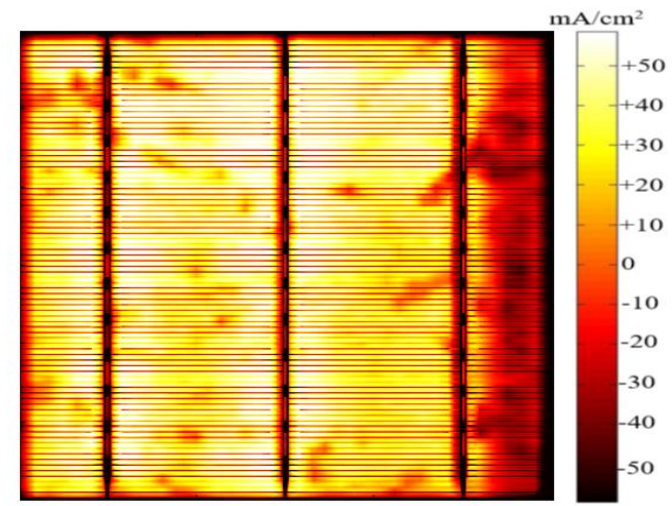

(a)

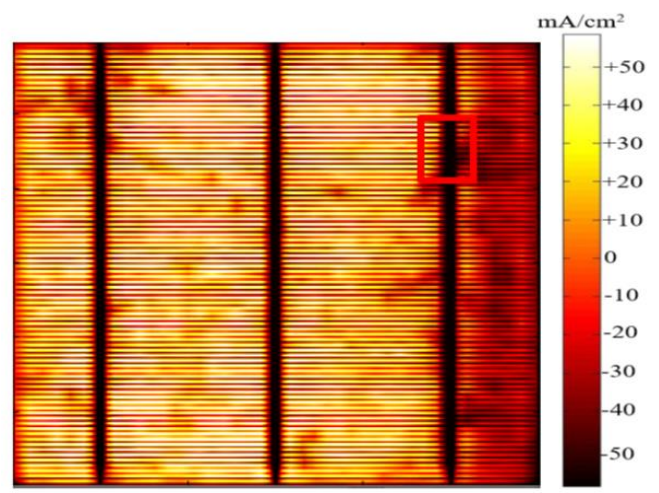

(b)

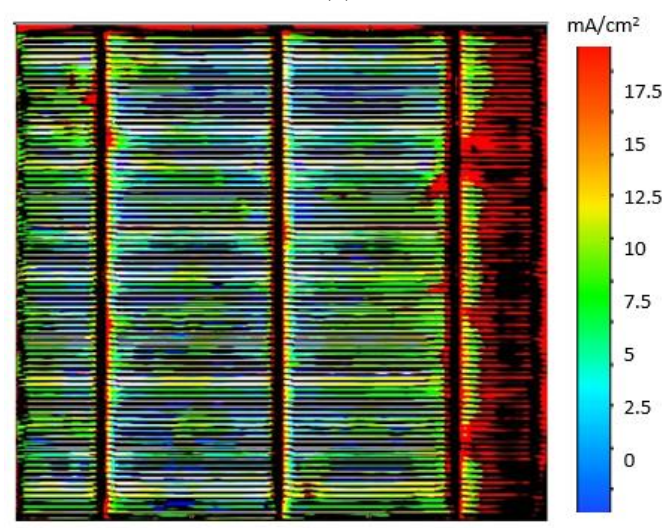

(c)

Fig. 7. $J_{s c}$ for Solar cell \#2. (a) before the PID test, (b) after the PID is completed, (c) Difference in the $J_{s c}$ before and after the PID test.
We have taken the SEM image of the labelled area in Fig 7(b) for further examination. The output image of the inspection is shown in Fig. 8(a). We have witnessed that after performing the PID test, the dark area (potential crack) damaged the busbar, this is evident by the front surface view.

In Fig. 8(b), we have observed at the back surface of the busbar. The PID test initiated further damage to the rear surface of the solar cell sample. There are a number of 'pits' in the contacts and material migration from the contact areas. As a result, we prove that it is likely to have a critical PID for a solar cell that comprises entirely/partially dark regions in the original EL image.
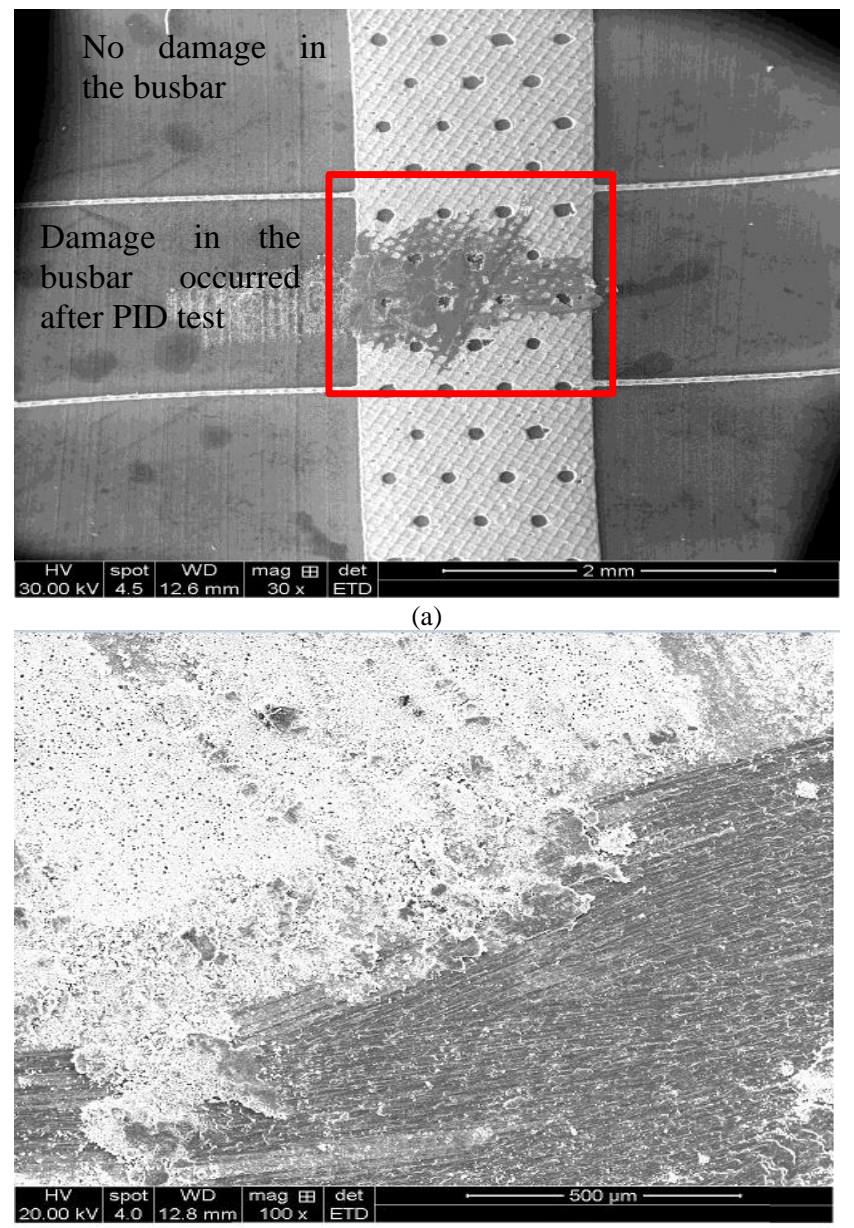

(b)

Fig. 8. SEM for solar cell \#2. (a) front surface, (b) Back surface.

Now, considering solar cell sample \#3 which was also imaged before and after PID testing. The $J_{s c}$ before the PID test is shown in Fig. 9(a), whilst after the PID test is performed, a large reduction in the $J_{s c}$ was observed, particularly in the dark area on the left-hand side of the EL image, and in the LBIC image, which is shown in Fig. 9(b).

The difference between the $J_{s c}$ before and after the PID test is shown in Fig. 9(c). We can remark that there is negative current (leakage) in the areas where the dark (crack) is present. This result reinforces our earlier conclusion that cracks can lead to greater decreases in current density, indicative of regions where the solar cell operation has completely broken down. Hence, hotspots are expected to appear these locations if the cell is operated under field conditions, which would lead to further degradation. 


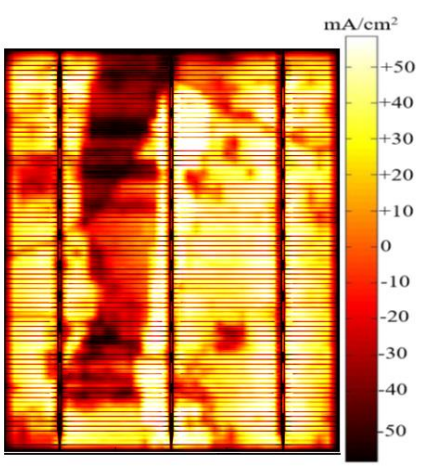

(a)

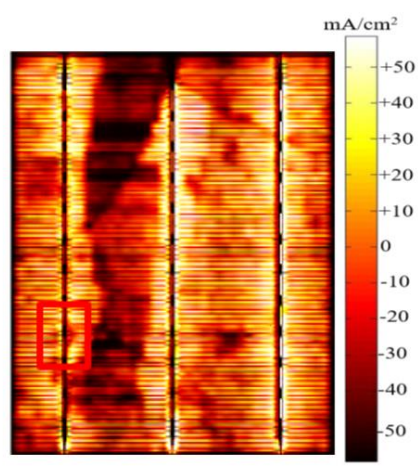

(b)

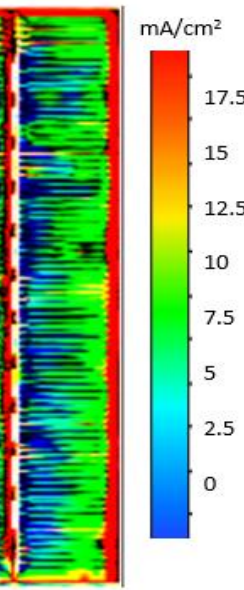

(c)
Fig. 9. $J_{s c}$ for Solar cell \#3. (a) before the PID test, (b) after the PID is completed, (c) Difference in the $J_{s c}$ before and after the PID test.

In contrast, we have further investigated the labelled area in Fig. 9(b), using an SEM. The result of the SEM inspection is displayed in Fig. 10. By inspection of the busbar's metallic (solder) joint to the solar cell surface, it is evident that the solder has migrated and deformed around the solder joint. This microscopic observation leads to understand why some solar cells, after PID tests, demonstrate significant decreases in the output current; clearly these changes lead to the development of hotspots and open circuits forming at the solder joints.

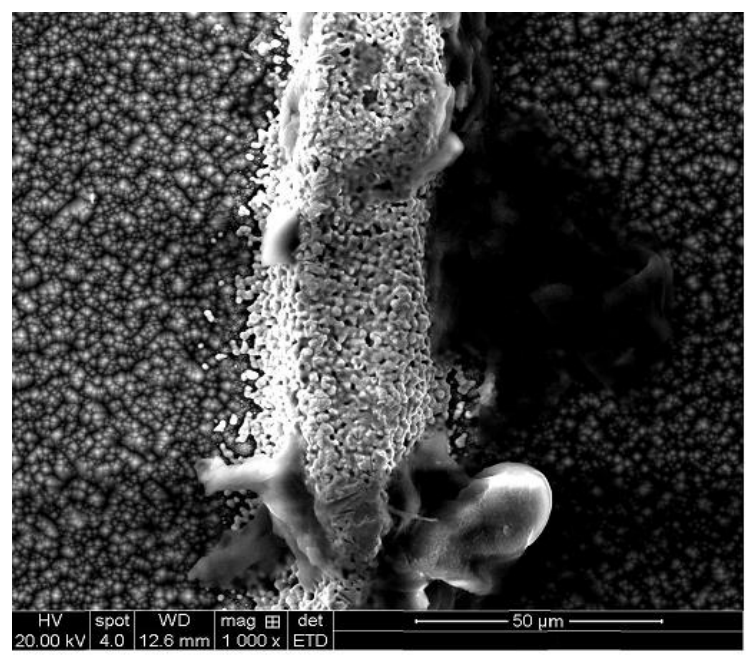

Fig. 10. SEM image for the front surface of the labelled area in Fig. 9(b).
As the solar cell exhibits significant losses in the $J_{s c}$ we have decided to test the cell under standard test conditions (illumination of $1000 \mathrm{~W} / \mathrm{m}^{2}$ and cell temperature 25 degrees), as shown in Fig. 11(a). The resultant current-voltage (J-V) curve compared with the theoretical values (taken from the solar cell datasheet) is shown in Fig. 11(b). The current density at short circuit conditions dropped by $20.5 \%$, calculated using (1), whereas $4.9 \%$ was the drop in the Voc calculated using (2). This concludes the fact that dark areas in EL images likely means that the cells are being impacted with severe PID.
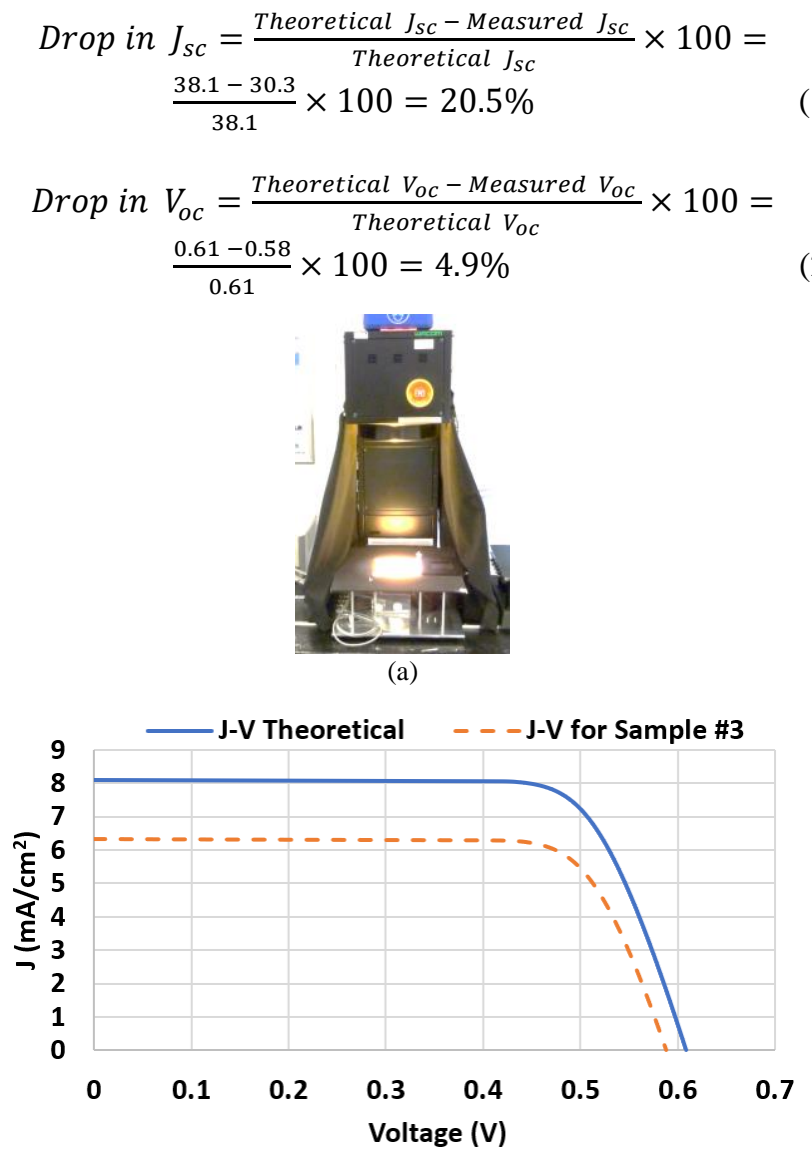

(b)

Fig. 11. (a) The solar cell is exposed to $1000 \mathrm{~W} / \mathrm{m}^{2}$ illumination, (b) Measured J-V curve under STC conditions.

In In Fig. 12(a), we show the results of the $J_{S C}$ for the last solar cell sample \#4, which exhibited the largest number of cracks at the outset. We can notice in the initial EL image that the black area represents a complete breakdown in the cell. After the PID test was completed, the image (Fig. 12(b)) confirms an expansion of the breakdown area in the cell (labelled as A1 and A2). In Fig. 12(c), the difference in the $J_{S C}$ before and after the PID test is presented. The expansion in the defect is evident indicating complete breakdown of $\sim 15 \%$ of the cell. The cause of this breakdown is likely to be due to localized heating of the silicon material leading to expansion and greater stress on the cracked surface. It is worth noting that, if the solar cell sample were still exposed in the field, these additional breakdown regions would appear after a longer period, for example after months of operation, due to the lower stress conditions. 


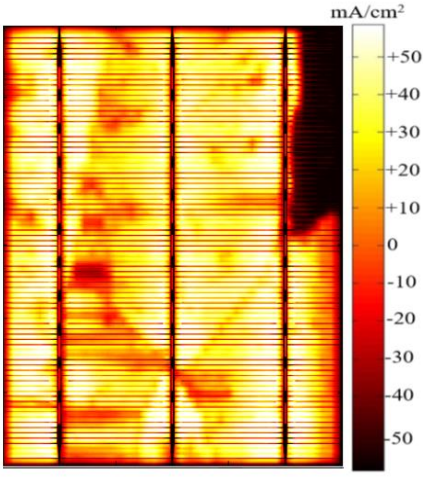

(a)

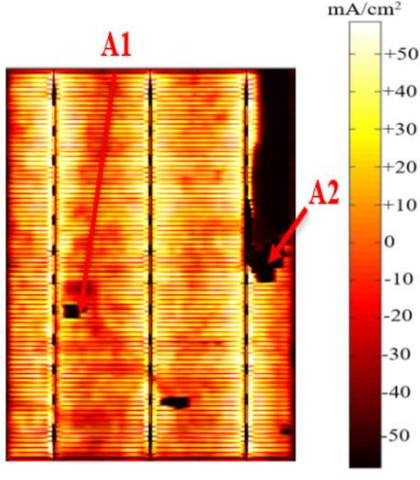

(b)

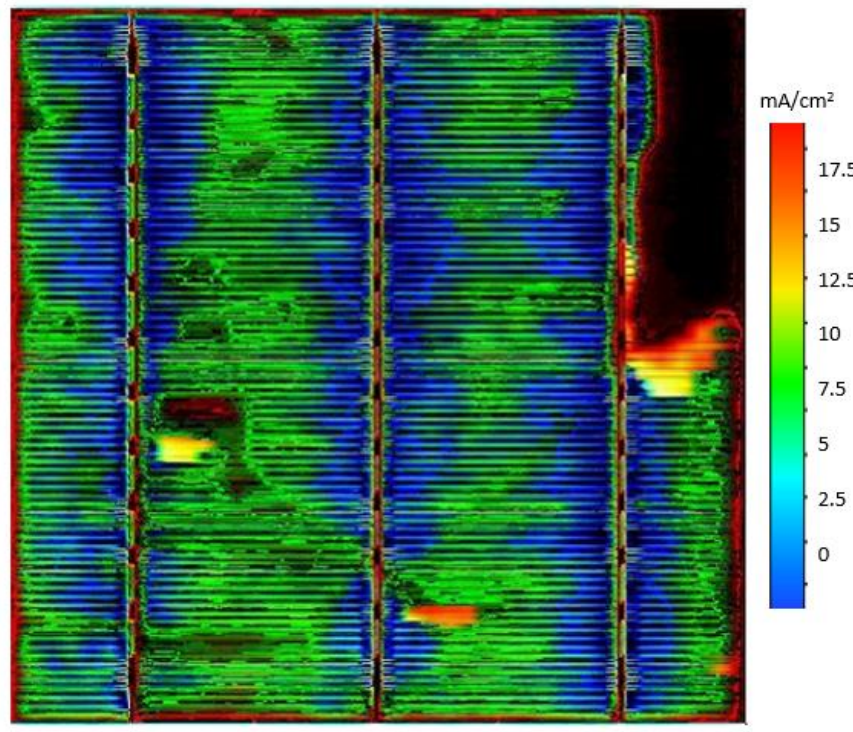

(c)

Fig. 12. $J_{s c}$ for Solar cell \#4. (a) before the PID test, (b) after the PID is completed, (c) Difference in the $J_{s c}$ before and after the PID test.

The breakdown areas were analyzed using a SEM. For example, the output SEM image for A1 is shown in Fig. 13(a). It appears that the crack has initiated a significant breakdown in the cell (the black area in the middle of Fig. 13(a)) and hundreds of similar potential breakdown blocks in the surrounding area. The white areas in the image represent are measured as Silicon using Energy-dispersive X-ray spectroscopy (EDX). In comparison, the black area corresponds to aluminum (Al), indicating that a large defect in the solar cell, and the solar cell rear contact (made of $\mathrm{Al}$ ) is only visible. Additionally, the SEM image for A2 is shown in Fig. 13(b); only 6\% silicon was found in this area, along with $7 \%$ silver (Ag) due to the expansion of the busbar material and $87 \%$ of Al. Accordingly, this part of the solar cell has a complete breakdown, not generating any photocurrent.

In reviewing the abovementioned observations, the breakdown area in the solar cell sample \#4 leads to a drop in the $J_{s c}$ after the PID test was completed and a further breakdown area adjacent to the initial breakdown. These observations suggest the following:

1) Breakdown in EL images are likely to span different breakdown regions after field operation.

2) Solar cells with this type of structural defects are indeed affected by PID.
To summarize the outcomes of this section, cracks in solar cells lead to an increase in PID and the size/shape of the initial defect has a strong bearing on the degraded cell. Our results indicate that minor cracks/fractures do not significantly degrade the solar cell under PID tests, which were conducted for 96 hours at $-160 \mathrm{~V}$. In stark contrast, larger cracks seen in dark areas of EL images resulted in severe PID. In addition, SEM imaging showed the formation of material defects; we have proved that cracks can result in further breakdown areas and a further reduction of the capacity of the busbars.
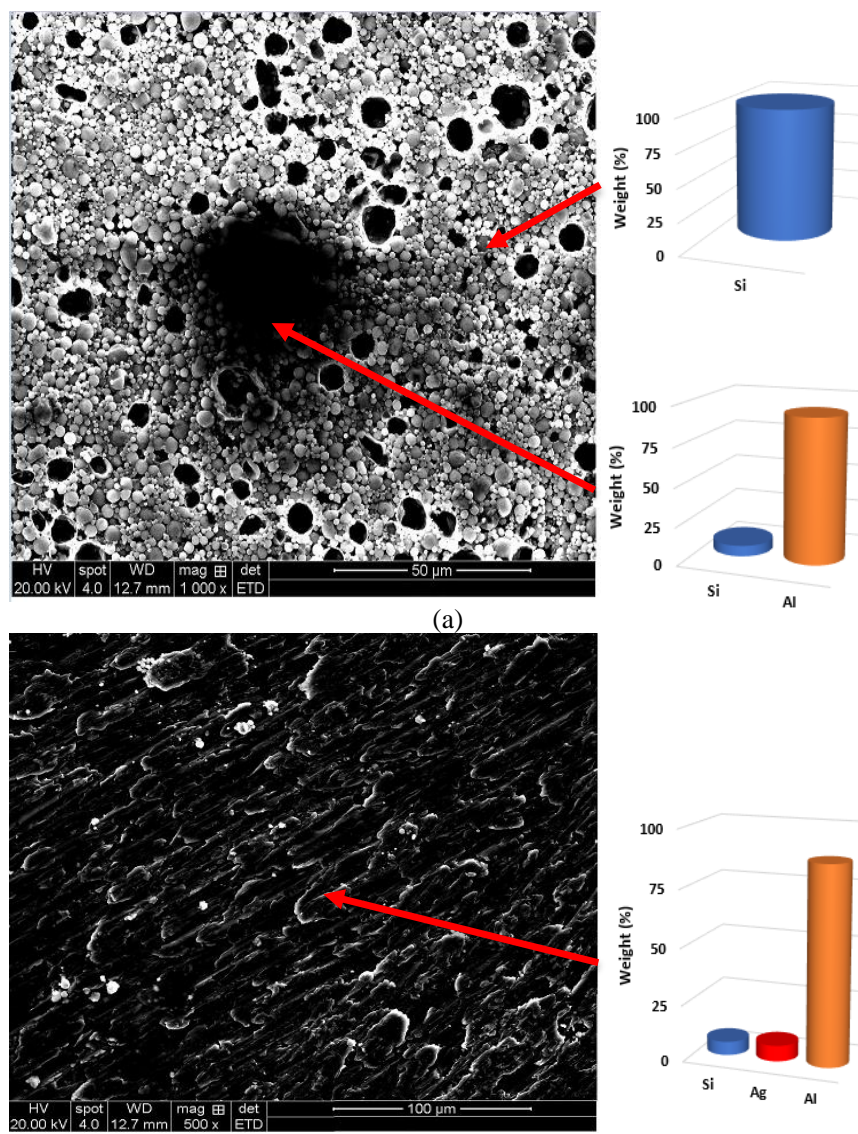

(b)

Fig. 13. (a) SEM image for the front surface of area A1, (b) SEM image for the front surface of area A2.

\section{OUtPut Power Measurements AND Thermal ANALYSIS}

The solar simulator was used to test the performance of the cells, and the output power at maximum power point vs irradiance is presented in Fig. 14(a). The irradiance ranges from 100 (0.1 Sun) to 1000 ( $1 \mathrm{Sun}) \mathrm{W} / \mathrm{m}^{2}$, while the cell's temperature was maintained at the standard test condition, $25^{\circ} \mathrm{C}$.

For sample \#1, affected by minor cracks, the cell generated more output power, with an average power output of $2.36 \mathrm{~W}$. Cells \#2 and \#3 show similar levels of cracking (as demonstrated by the dark areas in the EL image); so, their output averaged power at 1 Sun irradiance equals $1.81 \mathrm{~W}$ and 1.62 , respectively. Finally, the maximum drop in the averaged power was observed for the last solar cell sample \#4, which exhibited the most severe cracking and showed an output power of only $1.32 \mathrm{~W}$. 


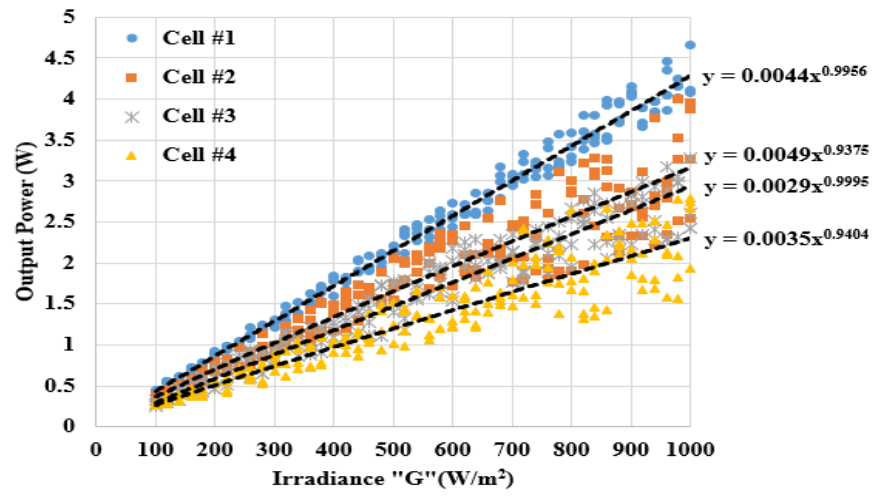

(a)

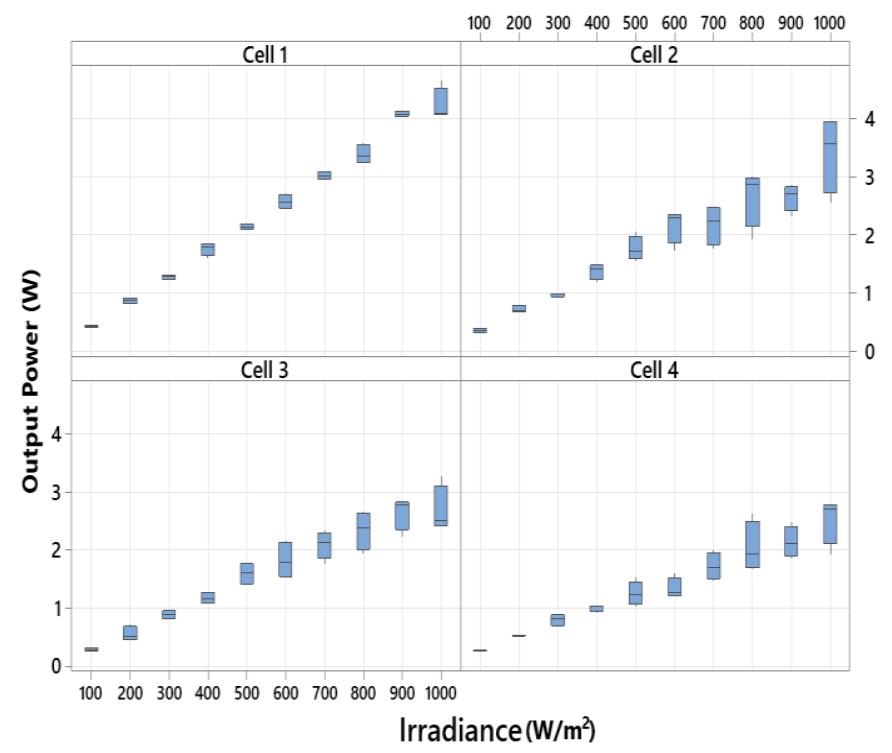

(b)

Fig. 14. (a) Output power of the cells under illumination from 100 to 1000 $\mathrm{W} / \mathrm{m}^{2}$, and cell temperature is 25 degrees, (b) Boxplot of the output power vs irradiance.

This data is supportive that as the crack size increases in solar cells, PID testing has a greater affect the output power performance. Furthermore, if we consider Fig. 14(b), the measured power has a more pronounced distribution away from the mean as the irradiance level increases. For example, the distribution of the measured output power at $1000 \mathrm{~W} / \mathrm{m}^{2}$ is $\pm 15 \%$ from the actual median value, while it is only equal to $\pm 2 \%$ at $100 \mathrm{~W} / \mathrm{m}^{2}$.

In addition to the electrical tests, thermal images of the solar cells have been captured using a thermal camera (FLIR C3-X) with thermal sensitivity of $0.07^{\circ} \mathrm{C}$. All the tested solar cells were connected with pure resistive load 1 ohms and illuminated under $1000 \mathrm{~W} / \mathrm{m}^{2}$. The cells were left for approximately 10 minutes to develop the hotspots.

The results of the experiment are presented in Fig. 15. We can remark that the cell's temperature of cell \#1 is uniformly distributed and at nearly STC condition, 25 degrees. For cell \#2 and cell \#3, it is evident that there is a localized increase in the temperature, which reaches as high as $40^{\circ} \mathrm{C}$ in the areas where a crack (dark) appears. Considering Fig. 15(c), we can recognise that in the middle busbar, there is a minor hotspot is now affecting the cell; this is because of the expansion of the crack after the PID was performed.
Because cell \#4 has the large breakdown region (as shown in the earlier EL image in Fig. 4), a rapid increase $\left(60^{\circ} \mathrm{C}\right)$ of the cell temperature is observed. This is because a reversebiased voltage is expected in this region, hence, a hotspot in the cell has now been developed. In summary, the main effects of the cracks themselves on hotspot temperatures are,

1) A larger crack (dark) will drive the affected cell considerably into reverse bias.

2) An increase of the shading effectively decreases the photo-illuminated region leading to a reduction in the cell current. These two effects run counter to each other and lead to the development of the hotspot.

To consolidate the results, we have determined the crack size of each tested solar cell using the EL and LBIC images and assigned them into four categories, 1.2-1.5\%, 3-4.5\%, 10$11.5 \%$, and $15-17 \%$. The degradation rate for each category has been calculated by dividing the measured power by the theoretical power and the results are presented in Fig. 16(a). According to the largest study to date on PV degradation, [19], the median degradation is $-0.5 \% / y e a r$ for a PV system, while for a standalone solar cell sample is $-1.0 \%$. Accordingly, in Fig. 16(a), we have labelled in "red" all calculated degradation rates that were observed below $-1.0 \%$. As a result, we found no significant degradation in the cell with $1.2-1.5 \%$ crack size, while for all other considered crack sizes, a significant degradation is observed.

Similarly, we observed the thermal performance (cells temperature) under varying solar irradiance (see Fig. 16(b)). The results show that for the crack sizes from $1.2-11.5 \%$, it is unlikely the cell develops a hotspot (nearly all measurements are below the standard baseline of $30^{\circ} \mathrm{C}$, IEC61215/61646). In contrast, if the crack size is in the range between $15-17 \%$, a hotspot is expected to develop and has an increase in the cell's temperature to approximately $60^{\circ} \mathrm{C}$.

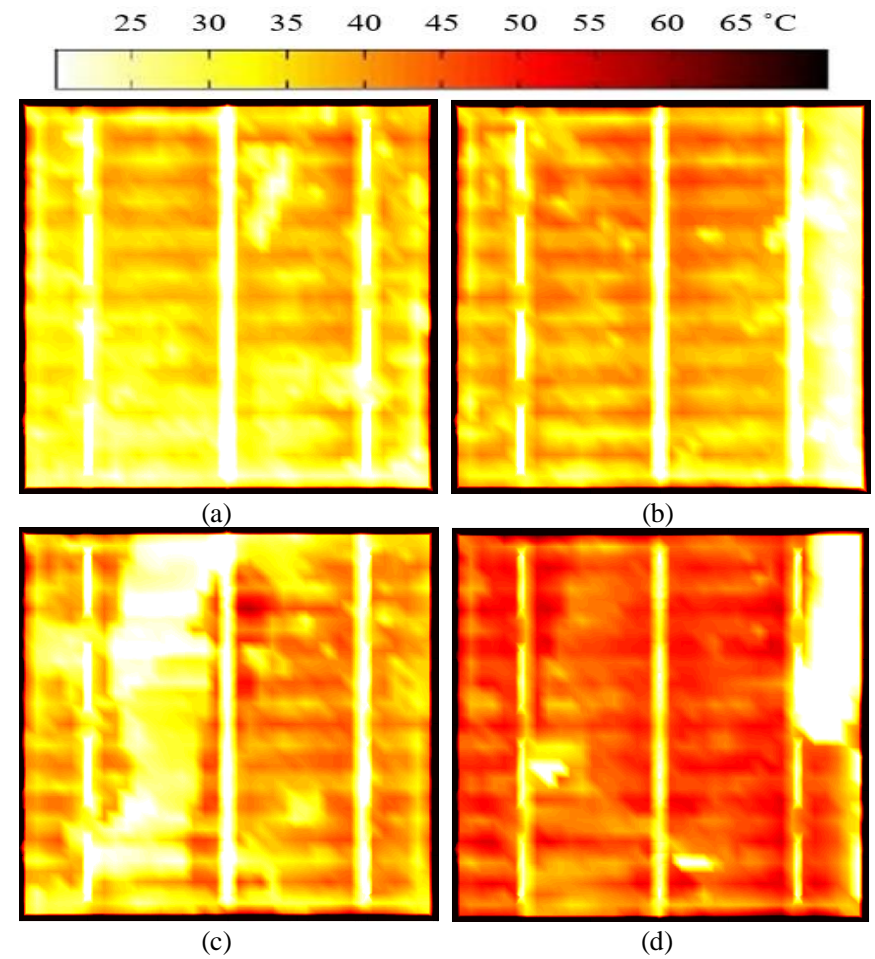

Fig. 15. Thermal image of the solar cell samples $(125 \times 125 \mathrm{~mm})$ at 1000 $\mathrm{W} / \mathrm{m}^{2}$. (a) Solar cell \#1, (b) Solar cell \#2, (c) Solar cell \#3, (d) Solar cell \#4. 


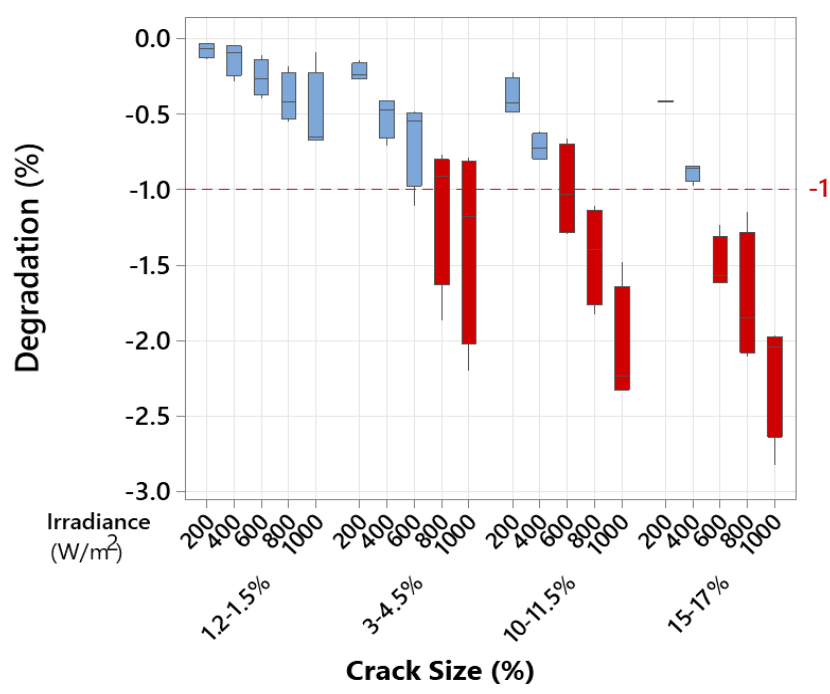

(a)

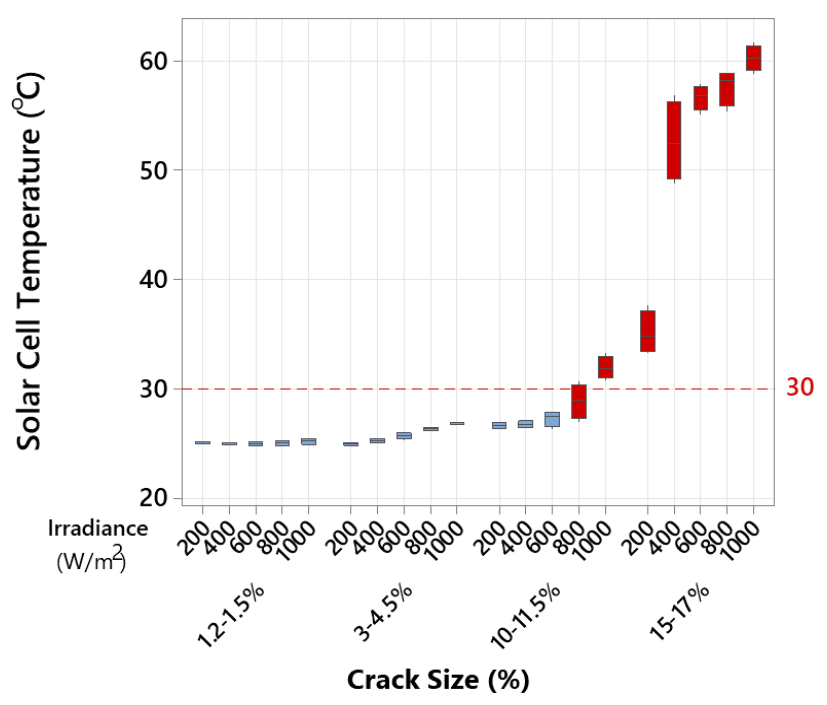

(b)

Fig. 16. Irradiance spectrum of the crack size vs. (a) Degradation, (b) Temperature.

\section{Conclusions}

This work reports the effect of cell cracks and structural defects on PID in solar cells. We have analyzed four solar cell samples with differing levels of cracks, including minor cracks, dark areas, and cells with large breakdown regions. It was found that minor cracks, represented by small spots and points in the EL image, has no considerable effect upon solar cell output and PID. However, larger cracks can lead to severe increases in PID in solar cell. The same outcome is recognized when testing a solar cell with a breakdown area. These results could be used to understand solar cells EL images taken in the field, which is now becoming an industry standard for quality and reliability checks. Therefore, if the EL image shows a dark area or breakdown regions, our results suggest that the PV module is likely to be affected by PID, resulting in a reduction in the current density, open-circuit voltage, and degrading its performance. We also confirmed the relationship between the cracks and the presence of hotspots. As the crack (dark) area increased, there was a further increase in the cell's temperature under standard test conditions.

\section{REFERENCES}

[1] K. Sporleder et al., "Potential-Induced Degradation of Bifacial PERC Solar Cells Under Illumination," in IEEE Journal of Photovoltaics, vol. 9, no. 6, pp. 1522-1525, Nov. 2019, doi: 10.1109/JPHOTOV.2019.2937231.

[2] M. Zhang, W. Zhou, R. Chen, M. Wong and H. Kwok, "Characterization of DC-Stress-Induced Degradation in Bridged-Grain Polycrystalline Silicon Thin-Film Transistors," in IEEE Transactions on Electron Devices, vol. 61, no. 9, pp. 3206-3212, Sept. 2014, doi: 10.1109/TED.2014.2341676.

[3] M. Xue, M. Wang, Z. Zhu, D. Zhang and M. Wong, "Degradation Behaviors of Metal-Induced Laterally Crystallized n-Type Polycrystalline Silicon ThinFilm Transistors Under DC Bias Stresses," in IEEE Transactions on Electron Devices, vol. 54, no. 2, pp. 225-232, Feb. 2007, doi: 10.1109/TED.2006.888723.

[4] Q. Wang, "Research on the effect of encapsulation material on anti-PID performance of $1500 \mathrm{~V}$ solar module," in Optik, vol. 202, pp. 163540, Feb. 2020, doi: 10.1016/j.ijleo.2019.163540.

[5] K. G. Bedrich et al., "Quantitative Electroluminescence Imaging Analysis for Performance Estimation of PID-Influenced PV Modules," in IEEE Journal of Photovoltaics, vol. 8, no. 5, pp. 1281-1288, Sept. 2018, doi: 10.1109/JPHOTOV.2018.2846665.

[6] H. Wang, P. Zhao, H. Yang, J. Chang, D. Song and S. Sang, "Performance variation of dark current density-voltage characteristics for PID-affected monocrystalline silicon solar modules from the field," in Microelectronics Reliability, vol. 81, pp. 320-327, Feb. 2018, doi: 10.1016/j.microrel.2017.11.003.

[7] K. Sporleder, M. Turek, N. Schüler, V. Naumann, D. Hevisov, C. Pöblau, S. Großer, H. Schulte-Huxel, J. Bauer and C. Hagendorf, "Quick test for reversible and irreversible PID of bifacial PERC solar cells," in Solar Energy Materials and Solar Cells, vol. 219, pp. 110755, Jan. 2021, doi: 10.1016/j.solmat.2020.110755.

[8] M. Dhimish and P. Mather, "Ultrafast High-Resolution Solar Cell Cracks Detection Process," in IEEE Transactions on Industrial Informatics, vol. 16, no. 7, pp. 4769-4777, July 2020, doi: 10.1109/TII.2019.2946210.

[9] M. Dhimish, V. d'Alessandro and S. Daliento, "Investigating the Impact of Cracks on Solar Cells Performance: Analysis based on Nonuniform and Uniform Crack Distributions," in IEEE Transactions on Industrial Informatics, doi: 10.1109/TII.2021.3088721.

[10] W. J. Ho, G. Y. Chen and J. J. Liu, "Enhancing Photovoltaic Performance of Plasmonic Silicon Solar Cells with ITO Nanoparticles Dispersed in SiO2 Anti-Reflective Layer," in Materials, vol. 12, no. 10, pp. 1614, May 2019, doi: doi.org/10.3390/ma12101614.

[11] M. Dhimish, Y. Hu, N. Schofield, and R. G. Vieira, "Mitigating PotentialInduced Degradation (PID) Using SiO2 ARC Layer," in Energies, vol. 13, no. 19 , pp. 5139, Oct. 2020, doi: doi.org/10.3390/en13195139.

[12] M. D. H. Chowdhury et al., "Effect of $\mathrm{SiO} 2$ and $\mathrm{SiO} 2 / \mathrm{SiNx}$ Passivation on the Stability of Amorphous Indium-Gallium Zinc-Oxide Thin-Film Transistors Under High Humidity," in IEEE Transactions on Electron Devices, vol. 62, no. 3, pp. 869-874, March 2015, doi: 10.1109/TED.2015.2392763.

[13] M. Dhimish, "Micro cracks distribution and power degradation of polycrystalline solar cells wafer: Observations constructed from the analysis of 4000 samples," in Renewable Energy, vol. 145, pp. 466-477, Jan. 2020, doi: 10.1016/j.renene.2019.06.057.

[14] M. R. U. Rahman and H. Chen, "Defects Inspection in Polycrystalline Solar Cells Electroluminescence Images Using Deep Learning," in IEEE Access, vol. 8, pp. 40547-40558, 2020, doi: 10.1109/ACCESS.2020.2976843.

[15] M. Dhimish and V. Holmes, "Solar cells micro crack detection technique using state-of-the-art electroluminescence imaging," in Journal of Science: Advanced Materials and Devices, vol. 4, no. 4, pp. 499-508, Dec. 2019, doi: 10.1016/j.jsamd.2019.10.004.

[16] J. Käsewieter, F. Haase and M. Köntges, "Model of Cracked Solar Cell Metallization Leading to Permanent Module Power Loss," in IEEE Journal of Photovoltaics, vol. 6, no. 1, pp. 28-33, Jan. 2016, doi: 10.1109/JPHOTOV.2015.2487829.

[17] F. Omnes, J. -. Guillaume, G. Nataf, D. Jager-Waldau, P. Vennegues and P. Gibart, "Substrate free GaAs photovoltaic cells on Pd-coated silicon with a 20\% AM1.5 efficiency," in IEEE Transactions on Electron Devices, vol. 43, no. 11, pp. 1806-1811, Nov. 1996, doi: 10.1109/16.543011.

[18] B. Du, R. Yang, Y. He, F. Wang and S. Huang, "Nondestructive inspection, testing and evaluation for Si-based, thin film and multi-junction solar cells: An overview," in Renewable and Sustainable Energy Reviews, vol. 78, pp. 1117-1151, Oct. 2017, doi: 10.1016/j.rser.2017.05.017.

[19] D. C. Jordan and S. R. Kurtz, "Photovoltaic degradation rates—an analytical review," in Progress in photovoltaics: Research and Applications, vol. 21, pp. 1, pp. 12-29, Oct. 2013, doi: 10.1002/pip.1182. 